\title{
Geospatial analyses identify regional hot spots of diffuse gastric cancer in rural Central America
}

\author{
Ricardo L. Dominguez ${ }^{1}$, Charlotte B. Cherry ${ }^{2}$, Dago Estevez-Ordonez ${ }^{3}$, Robertino Mera ${ }^{4}$, Veronica Escamilla ${ }^{5}$, \\ Michael Pawlita ${ }^{6}$, Tim Waterboer ${ }^{6}$, Keith T. Wilson ${ }^{4}$, Richard M. Peek ${ }^{4}$, Gloria Tavera ${ }^{7}$, Scott M. Williams ${ }^{7}$, \\ Margaret L. Gulley ${ }^{8}$, Michael Emch ${ }^{9}$ and Douglas R. Morgan $3,4,10^{*}$
}

\begin{abstract}
Background: Geospatial technology has facilitated the discovery of disease distributions and etiology and helped target prevention programs. Globally, gastric cancer is the leading infection-associated cancer, and third leading cause of cancer mortality worldwide, with marked geographic variation. Central and South America have a significant burden, particularly in the mountainous regions. In the context of an ongoing population-based casecontrol study in Central America, our aim was to examine the spatial epidemiology of gastric cancer subtypes and H. pylori virulence factors.
\end{abstract}

Methods: Patients diagnosed with gastric cancer from 2002 to 2013 in western Honduras were identified in the prospective gastric cancer registry at the principal district hospital. Diagnosis was based on endoscopy and confirmatory histopathology. Geospatial methods were applied using the ArcGIS v10.3.1 and SaTScan v9.4.2 platforms to examine regional distributions of the gastric cancer histologic subtypes (Lauren classification), and the H. pylori CagA virulence factor. Getis-Ord-Gi hot spot and Discrete Poisson SaTScan statistics, respectively, were used to explore spatial clustering at the village level (30-50 rural households), with standardization by each village's population. H. pylori and CagA serologic status was determined using the novel H. pylori multiplex assay (DKFZ, Germany).

Results: Three hundred seventy-eight incident cases met the inclusion criteria (mean age 63.7, male 66.3\%). Areas of higher gastric cancer incidence were identified. Significant spatial clustering of diffuse histology adenocarcinoma was revealed both by the Getis-Ord-Gl* hot spot analysis ( $P$-value $<0.0015$; range $0.00003-0.0014 ; 99 \% \mathrm{Cl}$ ), and by the SaTScan statistic (P-value < 0.006; range 0.0026-0.0054). The intestinal subtype was randomly distributed. $H$. pylori CagA had significant spatial clustering only in association with the diffuse histology cancer hot spot (GetisOrd-Gi* $P$ value $\leq 0.001$; range 0.0001-0.0010; SaTScan statistic $P$ value 0.0085). In the diffuse gastric cancer hot spot, the lowest age quartile range was $21-46$ years, significantly lower than the intestinal cancers $(P=0.024)$.

Conclusions: Geospatial methods have identified a significant cluster of incident diffuse type adenocarcinoma cases in rural Central America, suggest of a germline genetic association. Further genomic and geospatial analyses to identify potential spatial patterns of genetic, bacterial, and environmental risk factors may be informative.

Keywords: Gastric cancer, Diffuse gastric cancer, Germline mutations, H. pylori, Central America, CA-4, Honduras

\footnotetext{
* Correspondence: drmorgan@uabmc.edu

${ }^{3}$ Vanderbilt Ingram Cancer Center (VICC), Vanderbilt University Medical

Center, Nashville, USA

${ }^{4}$ Division of Gastroenterology, Hepatology, and Nutrition, Vanderbilt

University Medical Center, Nashville, USA

Full list of author information is available at the end of the article
}

(c) The Author(s). 2019 Open Access This article is distributed under the terms of the Creative Commons Attribution 4.0 International License (http://creativecommons.org/licenses/by/4.0/), which permits unrestricted use, distribution, and reproduction in any medium, provided you give appropriate credit to the original author(s) and the source, provide a link to the Creative Commons license, and indicate if changes were made. The Creative Commons Public Domain Dedication waiver (http://creativecommons.org/publicdomain/zero/1.0/) applies to the data made available in this article, unless otherwise stated. 


\section{Background}

Gastric adenocarcinoma is the leading global cause of infection-related cancer mortality and overall is the third leading cause of cancer death [1-4]. Approximately 1 million incident cases are projected annually, with the majority of incident cases observed in eastern Asia, Latin America, and eastern Europe. Nearly 70\% of global cancers now occur in low/middle income countries (LMICs), and seven cancers, including gastric cancer, account for $70 \%$ of the LMIC cancer mortality burden [2].

Gastric cancer has marked geographic variability, assessed at the regional, country, and within-country levels [5]. In Latin America, a significant burden of disease is concentrated in the mountainous regions along the Pacific littoral $[6,7]$, the gastric cancer "altitude enigma", and may represent host genetic variation. Helicobacter pylori (H. pylori) virulence factors, and dietary and environmental risk exposures also play a role in the rural mountain villages [6]. There is also evidence that disrupted host- $H$. pylori coevolution, with mismatch of respective genetic ancestries, may play a role in cancer predisposition in Latin America [8-10]. H. pylori is the most common chronic bacterial infection in the world, affecting half of the world's population. Infection prevalence ranges from 20 to $35 \%$ in high income countries to 60-90\% in LMICs [10-12]. H. pylori CagA has been shown to be an important virulence factor for disease progression to gastric adenocarcinoma [13-16]. The principal subsets of gastric adenocarcinoma per the Lauren histologic classification are intestinal and diffuse, and recent findings in The Cancer Genome Atlas (TCGA) NIH initiative confirm these subtypes [17-19].

Germline genetic associations may be present in 5-10\% of gastric adenocarcinoma [20]. Hereditary diffuse gastric cancer (HDGC) is uncommon, and primarily driven by CDH1 mutations [20]. Recent studies suggest that homologous recombination (HR) germline mutations (PALB2, $B R C A 1, R A D 51 C$ ) are also important in familial clusters, including in Latin America [21]. Some familial clustering may also be attributable to shared bacterial or environmental exposures. Globally, intestinal gastric cancer is more common than diffuse by a $4.6: 2$ ratio [19], although in Central America and in Hispanics in the U.S., the diffuse subtype has a higher prevalence [22].

Geospatial methods and the use of geographic information systems (GIS) can delineate disease distributions and etiology, as well as inform prevention programs, however, few studies have applied spatial techniques to examine gastric cancer [23-26]. The objective of this study was to utilize geospatial methods to examine the spatial distributions of gastric cancer subtypes, in the context of an ongoing population-based, case-control study in Central America. The identified clusters of high gastric cancer incidence may implicate germline genetic associations, along with bacterial, dietary, or environmental co-factors.

\section{Methods \\ Study design and setting}

We performed spatial cluster analyses in the context of an active population-based, case-control study centered in western Honduras. The study was set in rural Honduras and is representative of the Central America Four ("CA-4") region (Guatemala, Honduras, El Salvador, Nicaragua), the largest LMIC region in the western hemisphere, with over 36 million inhabitants [26]. This mountainous region has a racial-ethnic mixture of primarily Hispanic Mestizo (95\%) and has among the highest gastric cancer incidence rates in the western hemisphere, with a high prevalence of $H$. pylori infection of over $80 \%$ [2, 27-29].

We prospectively identified all incident cases of gastric cancer between 2002 and 2013 from a registry within the Ministry of Health district hospital (Hospital de Occidente) of western Honduras in Santa Rosa de Copán, that serves as the principal referral center for the region. The hospital catchment area has been previously described [28]. The diagnosis of gastric cancer was based on endoscopic appearance and confirmatory histopathology. Western Honduras includes all or part of the three western states (departamentos) of Honduras, spanning $5000 \mathrm{~km}^{2}$, with an adult population of approximately 400,000. Each state is comprised of counties (municipalities) consisting of villages (aldeas) of 30-50 households on average, each with a unique geocode. Incident cases in the villages of the three western states in the referral area were included in the analysis. The crude incidence rates for each village were calculated for GIS mapping: number of cases per village population per 100,000 persons for the overall study period. The population data for each village was obtained from the Honduras Census Institution (Instituto Nacional de Estadistica) from the census year 2001 [30].

\section{H. pylori infection assessment}

H. pylori and CagA status were determined by the novel validated $H$. pylori multiplex serology. This antibody detection technology uses $15 \mathrm{H}$. pylori proteins bacterially expressed in full length as recombinant proteins in fusion with N-terminal glutathione-S-transferase (GST) and C-terminal a small tagging epitope (tag). Each GST-X-tag fusion protein was bound and affinity-purified on a different bead set with glutathione surface and marked with a distinct internal fluorescent color (SeroMap, Luminex Corp., Austin, TX, USA) [3136]. Seropositivity against each of the $15 \mathrm{H}$.pylori antigens, including CagA, was defined based on antigen-specific cut-point values, previously determined 
Table 1 Demographic and exposure factors of the intestinal and diffuse type gastric cancer cases

\begin{tabular}{|c|c|c|c|c|}
\hline Characteristics & Overall population & Spatial analysis cases & Cases without village geocodes ${ }^{b}$ & $P$ value \\
\hline Cancer Cases (N) & 498 & 378 & 120 & 0.047 \\
\hline Intestinal subtype & $259(52.0 \%)$ & $187(49.5)$ & $72(60)$ & \\
\hline Diffuse subtype & $239(48.0 \%)$ & $191(50.5)$ & $48(40)$ & \\
\hline Age, mean (SD) & $63.2(13.8)$ & $62.6(13.9)$ & $65.4(13.6)$ & 0.054 \\
\hline Gender & & & & 0.51 \\
\hline Female (\%) & $168(33.7)$ & $131(34.7)$ & $37(30.8)$ & \\
\hline Male (\%) & $330(66.3)$ & $247(65.3)$ & $83(69.2)$ & \\
\hline Family history GC (\%) & & & & 0.81 \\
\hline Yes (\%) & $33(6.6)$ & $26(6.9)$ & $7(5.8)$ & \\
\hline No (\%) & $448(90.0)$ & $340(89.9)$ & $108(90.0)$ & \\
\hline Not reported (\%) & $17(3.4)$ & $12(3.2)$ & $5(4.2)$ & \\
\hline Alcohol history (ever) & & & & 0.72 \\
\hline Yes (\%) & $107(21.8)$ & $79(21.4)$ & $28(23.3)$ & \\
\hline No (\%) & $373(76.1)$ & $284(76.8)$ & $89(74.2)$ & \\
\hline Not reported (\%) & $18(3.6)$ & $15(4.0)$ & $3(2.5)$ & \\
\hline Smoking history (ever) & & & & 0.72 \\
\hline Yes (\%) & $126(24.1)$ & $97(26.6)$ & $29(24.8)$ & \\
\hline No (\%) & 355 (73.6) & $267(73.2)$ & $88(75.2)$ & \\
\hline Not reported (\%) & $17(3.4)$ & $14(3.7)$ & $3(2.5)$ & \\
\hline H. pylori serodiagnosis ${ }^{a}$ & 385 & 286 & 99 & 0.82 \\
\hline Positive N (\%) & $337(87.5)$ & $251(87.8)$ & $86(86.9)$ & \\
\hline Negative N (\%) & $48(12.5)$ & $35(12.2)$ & $13(13.1)$ & \\
\hline H. pylori CagA antibodies ${ }^{a}$ & 385 & 286 & 99 & 0.64 \\
\hline Positive N (\%) & 361 (93.8) & $267(93.4)$ & $94(95.0)$ & \\
\hline Negative N (\%) & $24(6.2)$ & $19(6.6)$ & $5(5.0)$ & \\
\hline
\end{tabular}

${ }^{a}$ The comparison $P$ values refer to the spatial analysis cases with geocodes versus the excluded cases without the village-level (aldea) geocodes. ${ }^{b}$ In the initial study period, geocodes were at times limited to the municipality-level, without village-level data

${ }^{\mathrm{a}} \mathrm{H}$. pylori and CagA multiplex assay data were not available for all subjects in the study populations

in validation studies [29]. Positive H. pylori status was defined as seropositivity against more than 3 of these antigens.

\section{Geospatial and statistical analysis}

The Getis Ord Gi* local spatial cluster analysis method was implemented in $\operatorname{ArcGIS}^{\circ}$ version 10.3.1 (ESRI, Redlands, CA, USA) [37]. This method analyzes each feature in the context of its neighboring features (e.g., villages). A village (aldea) with a high value surrounded by other villages with high values may be a statistically significant hot spot. A fixed distance band of $23 \mathrm{~km}$ ( 14 miles) was used to examine spatial relationships of aldeas. We selected a $23 \mathrm{~km}$ distance to ensure that each aldea had multiple neighbors. The local sum of cases for each aldea and its neighbors is compared proportionally to the sum of all cases in the study area. If the local sum is very different from the expected local sum (and is too large to be random) it is a statistically significant hot (or cold spot).
Cluster $P$ values were adjusted for multiple testing using the False Discovery Rate (FDR) correction. Kulldorff's spatial scan statistic, a complementary methology, was used to validate the hot spot analysis results using SaTScan 9.4 (Boston, MA) [38]. A discrete Poisson model was used to identify high gastric cancer incidence clusters. The spatial scan statistic uses a circular roving window varying in size, that increases incrementally to encompass a maximum percent of the population $(25,50 \%$, etc.). We set the maximum window size to encompass up to $25 \%$ of the population. Counts that are higher than expected relative to the underlying population and study area are designated as a cluster. Cluster significance was determined using Monte Carlo simulation (999 permutations).

Diffuse histology cancer cases and intestinal histology cancer cases were analyzed separately. Cases of mixed or indeterminate histology were excluded from the geospatial analysis. The H. pylori CagA spatial distribution was analyzed subsequently. Gastric cancer cases without 


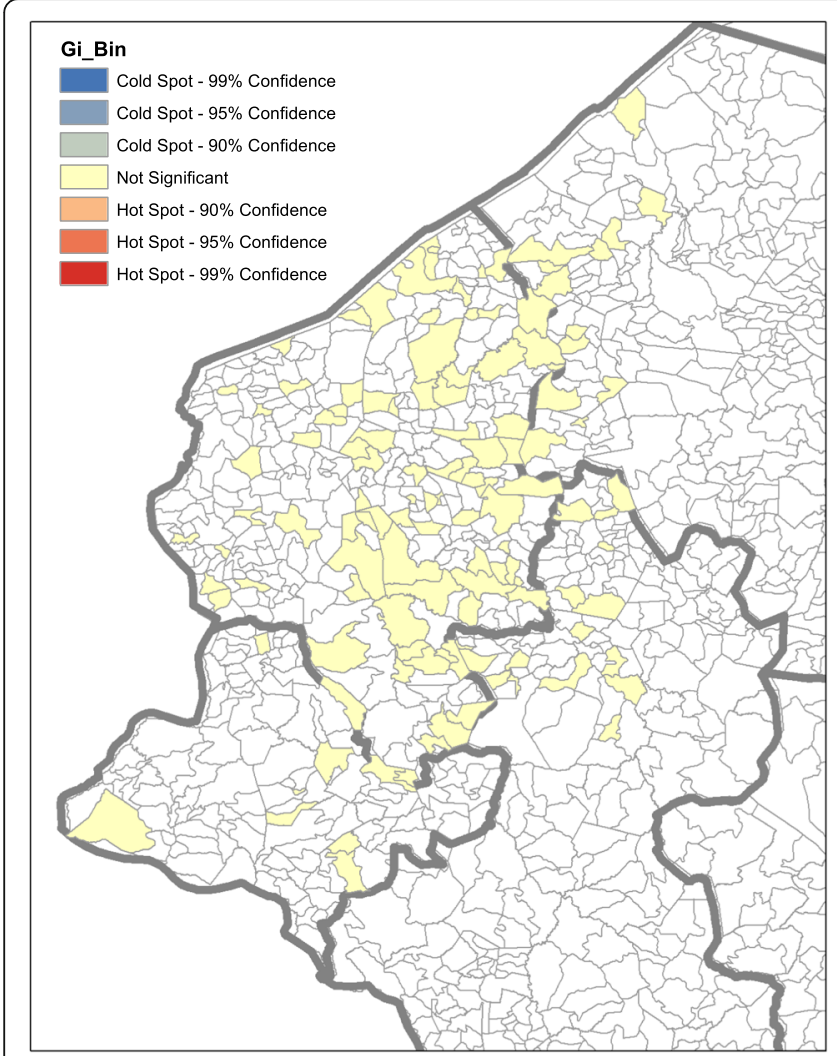

\section{Intestinal Gastric Cancer}

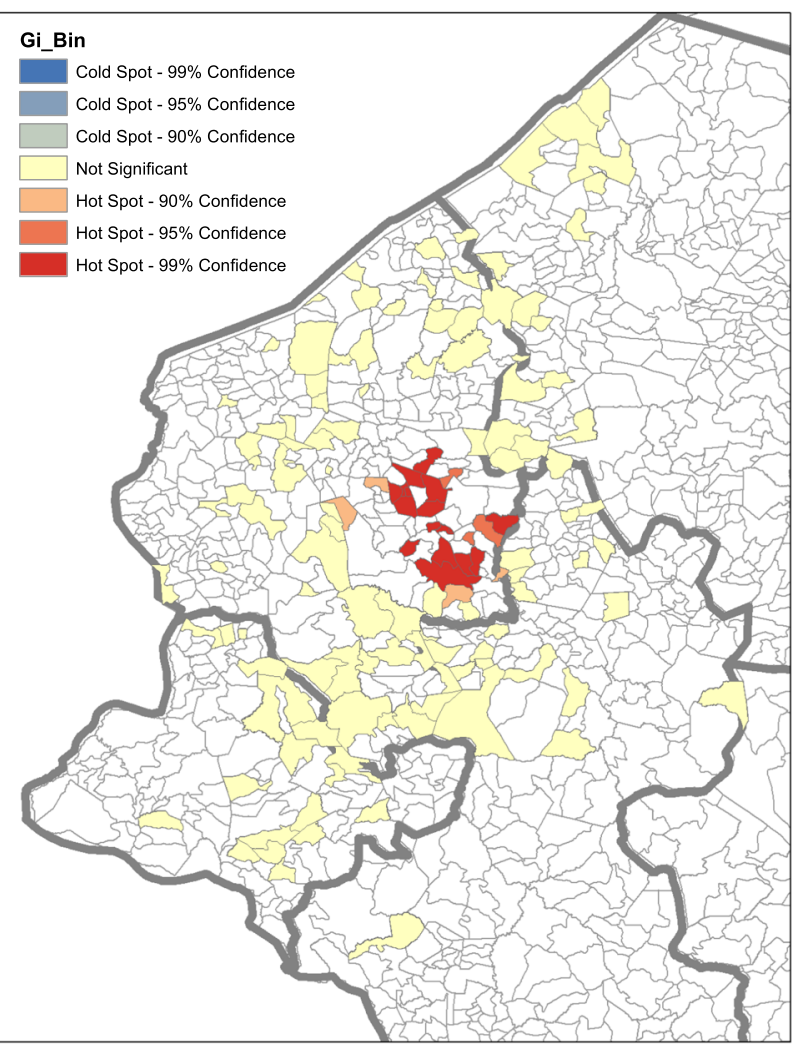

\section{Diffuse Gastric Cancer}

Fig. 1 Spatial clustering of diffuse type gastric cancer, Getis Ord Gi* hot spot cluster analysis. Spatial clustering of diffuse gastric cancer incident cases were identified by two independent GIS methodologies. The Getis Ord Gi* hot spot analysis (Fig. 1), identified three neighboring hotspots in western Honduras that may be considered one cluster area ( $P$ value $<0.0015$; range 0.00003-0.0014; $99 \%$ CI). The spatial scan statistic SaTScan (not shown) also demonstrated a statistically significant cluster (32 km radius) in the same location ( $P$-value $<0.006$; range $0.0026-0.0054)$. The intestinal subtype cancers were randomly distributed, and without high incidence clusters

aldea geocodes and aldea population data, were excluded from the geospatial analysis, by necessity, but included in the overall descriptive analyses of the study population. Demographic and clinical data were analyzed by chi-squared and univariable multinomial logistic regression analyses.

\section{Results}

A total of 702 gastric cancer patients were identified in the western Honduras gastric cancer registry from 2002 to 2013. There were 498 subjects with either intestinal or diffuse subtypes, with a mean age of 63.2 (SD 13.8), and $66.3 \%$ males $(n=330)$. Three hundred seventy-eight patients had validated village-level (aldea) geocode data for the spatial analysis. (Table 1). The subjects without village-level geocodes were primarily from the early study period, which primarily focused on county-level geocoding. The excluded subjects $(n=204)$ were from outside of the district hospital catchment area $(n=108)$, with non- intestinal/diffuse histology $(n=80$; mixed 42; indeterminate 35 ; other 3$)$, or had missing data $(n=16)$.
In the overall study population, there were 259 cases (52\%) of the intestinal subtype. Over three quarters of cancer patients $(87.5 \%)$ were positive for $H$. pylori infection by multiplex serology, of whom $93.8 \%$ were CagA positive. In this setting, tobacco and alcohol use was limited, with proportions of never-used of 73.6 and $76.1 \%$, respectively. A family history of gastric cancer was only noted in $6.6 \%$ of cases. No significant differences in demographic or clinical features were noted between the geospatial analysis study group (with aldea geocodes) and the subject group without geocodes, with the exception of borderline differences $(P=0.05)$ in age and proportions of the Lauren classification subtypes.

In the study period, in the catchment area, incident cases were identified in 219 villages within a total of 58 counties. Hot spots of diffuse gastric cancer incidence were identified by both independent methodologies (Fig. 1). The Getis Ord Gi* hot spot analysis identified three neighboring hotspots in western Honduras ( $P$ value $<0.0015$; range $0.00003-0.0014 ; 99 \% \mathrm{CI}$ ) that may be considered one cluster area. The spatial scan statistic 


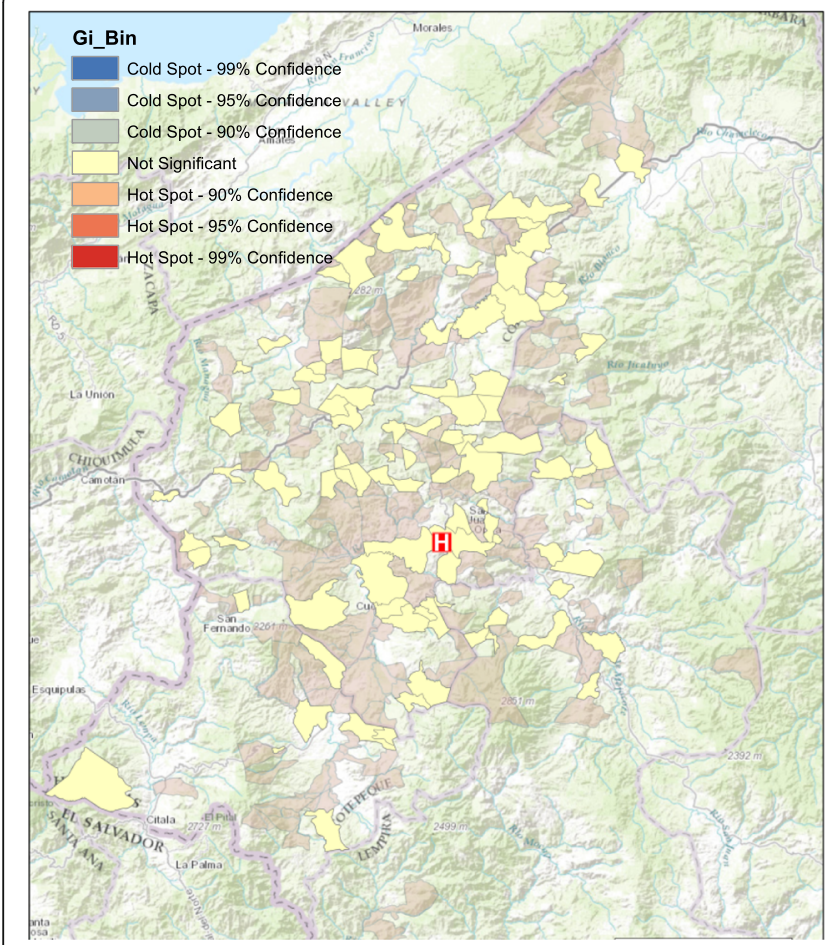

\section{Intestinal Gastric Cancer}

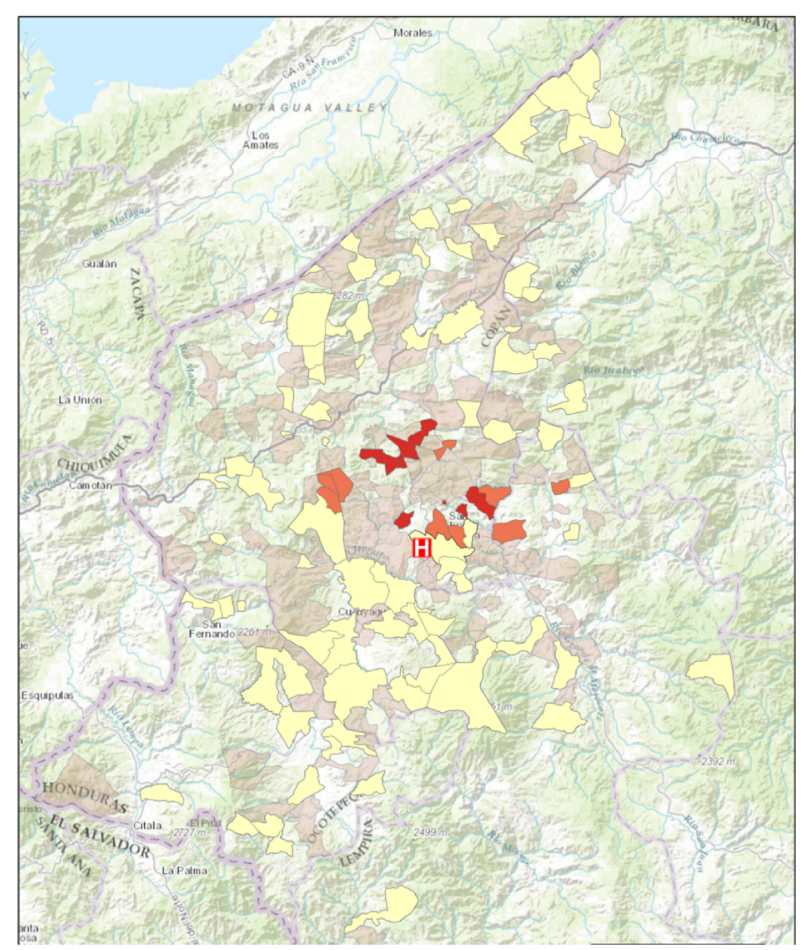

Diffuse Gastric Cancer

Fig. 2 Spatial analysis of H. pylori CagA, by Getis Ord Gi* hot spot cluster analysis. Clusters with a higher relative number of cases with $H_{\text {. pylori }}$ CagA infection were identified, but only in association with the incident diffuse cancer clusters. CagA hotspots were detected using the Getis-Ord $\mathrm{Gi}^{*}$ statistic $(P$ value $\leq 0.001$; range $0.0001-0.0010)$, as shown in Fig. 2. The H. pylori CagA hotspots was also observed with the SaTScan statistic $(P$ value $<0.0085)$ in the same area (not shown). This indicates that CagA may be a co-factor in the diffuse gastric cancer cluster area. CagA was randomly distributed among the diffuse cancers outside of the hotspot. CagA was also randomly distributed among the intestinal cancers in the hotspot area and in the western Honduras as a whole

results corroborated the hotspot findings. A single statistically significant cluster with a $32 \mathrm{~km}$ radius was identified in the same location as the hotspots using SaTScan ( $P$-value <0.006; range 0.0026-0.0054). The intestinal subtype cancers were randomly distributed, and without high incidence clusters. (Fig. 1).

Clusters of aldeas with a higher relative number of cases of $H$. pylori CagA were identified, but only among the diffuse type cancer hotspot clusters. Hotspots were detected using the Getis-Ord $\mathrm{Gi}^{*}$ statistic ( $P$ value $\leq 0.001$; range 0.0001-0.0010). (Fig. 2). The location of the H. pylori CagA hotspots was also observed with the SaTScan statistic, with a significant cluster $(P$-value $<$ 0.0085 ) with a $4 \mathrm{~km}$ radius, located in the same area. The overlap of the clustering areas of diffuse cancer and CagA areas was noted, indicating that CagA may be a co-factor in the area where germline mutations may be important. CagA was randomly distributed outside of the diffuse cancer cluster hotspot, and also among the intestinal cancers within this hotspot area.

We examined differences in demographic and exposure factors between cases that were located in the diffuse hotspot versus cases that were intestinal as well as all cancers outside of the cluster area. Table 2 summarizes the demographic and exposure factors of the hot spot (cluster) of diffuse gastric cancer for the 378 patients for which village-level geocodes were available. $H$. pylori multiplex serology data was available for 286 out of the 378 cases in the geospatial analysis. Importantly, diffuse cases in the diffuse gastric cancer hot spot had the lowest age interquartile $0-25 \%$ range, $21-46$ years, and were significantly younger than the intestinal cases located within the diffuse cluster $(P=0.024)$. The modest number of cases within the diffuse cancer hot spot may have limited the power to detect some significant differences in other risk exposures.

\section{Discussion}

Gastric cancer, the leading infection-associated cancer, demonstrates remarkable geographic variability. In Latin America, the burden is concentrated in the Pacific littoral mountainous regions of Mexico, Central America, and the Andes [6]. We have identified hot spots of the diffuse gastric cancer subtype, in the mountainous region of western Honduras, with rigorous geospatial 
Table 2 Demographic and exposure factors of the hot spot (cluster) of diffuse gastric cancer cases

\begin{tabular}{|c|c|c|c|c|}
\hline Characteristics & Cluster, Diffuse & Cluster, Intestinal & Non-cluster, Diffuse & Non-cluster, Intestinal \\
\hline $\begin{array}{l}\text { Geospatial methods }{ }^{\mathrm{a}} \text {, P-values } \\
\text { - Getis Ord Gi } \\
\text { - SaTScan }\end{array}$ & $\begin{array}{l}P<0.0015,99 \% C l \\
P<0.006\end{array}$ & Referent & Referent & Referent \\
\hline Cases (N) & 52 & 32 & 139 & 155 \\
\hline Histology, signet ring & $75 \%$ & na & $82 \%$ & na \\
\hline Age, mean (SD) & $60.2(16.5)$ & $69.7(9.9)$ & $61.3(14)$ & $63(13.1)$ \\
\hline Age IQR & $46-73$ & $62-76$ & $52-72$ & $53-73$ \\
\hline Age IQR 0-25\% & $21-46$ & $53-62$ & $23-52$ & $30-53$ \\
\hline$P$-values & Referent & $p=0.002$ & $p=0.61$ & $p=0.034$ \\
\hline \multicolumn{5}{|l|}{ Gender } \\
\hline Female N (\%) & $15(28.9)$ & $11(34.4)$ & $50(36.0)$ & $55(35.5)$ \\
\hline Male N (\%) & $37(71.1)$ & $21(65.6)$ & $89(64.0)$ & $100(64.5)$ \\
\hline$P$-values & Referent & $p=0.59$ & $p=0.36$ & $p=0.38$ \\
\hline Family history ${ }^{\mathrm{a}}(\mathrm{N})$ & 51 & 28 & 136 & 151 \\
\hline Yes (\%) & $2(3.9)$ & $3(10.7)$ & $7(5.2)$ & $14(9.3)$ \\
\hline No (\%) & $49(96.1)$ & $25(89.3)$ & $129(94.9)$ & $137(90.7)$ \\
\hline$P$-values & Referent & $p=0.25$ & $p=0.73$ & $p=0.24$ \\
\hline Alcohol history, ever (N) & 50 & 31 & 134 & 148 \\
\hline Yes (\%) & $13(26)$ & $10(32.3)$ & $25(18.7)$ & $31(21)$ \\
\hline No (\%) & $37(74)$ & $21(67.7)$ & $109(81.3)$ & $117(79)$ \\
\hline P-values & Referent & $p=0.54$ & $p=0.28$ & $p=0.46$ \\
\hline Smoking history, ever (N) & 50 & 31 & 134 & 149 \\
\hline Yes (\%) & $13(26)$ & $13(42)$ & $34(25.4)$ & $37(24.8)$ \\
\hline No (\%) & $37(74)$ & $18(58)$ & $100(74.6)$ & $112(75.2)$ \\
\hline$P$-values & Referent & $p=0.14$ & $p=0.93$ & $p=0.87$ \\
\hline H. pylori serodiagnosis $(\mathrm{N})$ & 38 & 25 & 107 & 116 \\
\hline Positive N (\%) & $31(81.6)$ & $20(80)$ & $96(89.7)$ & $104(89.7)$ \\
\hline Negative N (\%) & $7(18.4)$ & $5(20)$ & $11(10.3)$ & $12(10.3)$ \\
\hline$P$-values & Referent & $p=0.88$ & $p=0.20$ & $p=0.195$ \\
\hline H. pylori Cag A antibodies ${ }^{\mathrm{a}}(\mathrm{N})$ & 38 & 25 & 107 & 116 \\
\hline Positive (\%) & $33(86.8)$ & $22(88)$ & $100(93.5)$ & $112(96.5)$ \\
\hline Negative (\%) & $5(13.2)$ & $3(12)$ & $7(6.5)$ & $4(3.5)$ \\
\hline$P$-values & Referent & $p=0.89$ & $p=0.21$ & $p=0.039$ \\
\hline
\end{tabular}

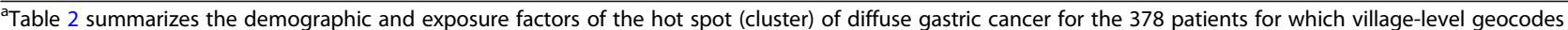
were available. In the initial study period, geocodes often limited to the municipality-level, without village-level data. H. pylori CagA multiplex serology data was available for 286 out of the 378 cases in geospatial analysis

${ }^{a}$ The cluster detection methods identify areas with high prevalence villages adjacent to other high prevalence villages. Therefore, while the prevalence is higher outside of the cluster, those patterns of higher incidence appear to be random

methods. We postulate that the geographic clustering and younger age of the diffuse gastric cancer patients may implicate a germline genetic association. This may represent a cluster of subjects with germline mutations of $C D H 1$, another tumor suppressor gene, or other recently described associations [20,39-42].

The cluster of diffuse adenocarcinomas may be due to several factors, including germline mutations, and may represent a Founder and/or endogamy effects. Kaurah et.al., observed a combination of both a Founder effect and endogamy influences in hereditary diffuse gastric cancer (HDGC) in rural British Colombia [43-45].
While all of the Central American populations originally derived from Asian migrations, it is possible that they were founded by different sub-groups, a migration Founder effect. This could be tested using large-scale genomic data. Similarly, following Spanish colonization there were substantial bottlenecks across Central America with high mortality due to newly introduced infections (eg. smallpox), a potential extinction Founder effect. The patterns of diffuse cancer may be affected by varying degrees of host bottlenecks across our sites. Lastly, endogamy may affect genetic risk in the isolated mountain villages, either due to mating patterns, 
patterns or the small effective population sizes. In the latter case, we would predict that the clusters would have smaller village population sizes.

The familial clustering in $5-10 \%$ of gastric cancer cases may be partially attributed to shared $H$. pylori virulence factors, as well as environmental, dietary, and behavioral factors [20]. Specific H. pylori virulence factors may cluster in families and populations. The CagA clusters in our analysis are directly linked with the diffuse gastric cancer cluster. This is likely a secondary association and co-factor with the postulated germline genetic association, given the lower overall CagA prevalence within the cluster area, as well as the younger age of the diffuse cancer patients. It could also be postulated that patterns of host and pathogen genetic variation differ by region, thereby disrupting a co-evolutionary history as we have previously demonstrated in Colombia [8]. Environmental factors may be important in some areas. For instance, volcanic soils have been proposed as a contributory factor in the Middle East [24]. Lastly, EBV infection accounts for $10 \%$ of global gastric cancer [19]. In Honduras, we have noted a prevalence of 9\%, without apparent geographic variation [46]. Polymorphisms in the EBV viral genome may impact oncogenicity, and warrant investigation of potential spatial patterns. Some EBV genome variants encode epitopes affecting innate or adaptive $\mathrm{T}$ cell response, implying that virulence factors may alter normal mechanisms controlling virus-induced cell growth $[47,48]$. In sum, interactions between $H$. pylori, EBV and other components of the microbiome may also play a role in regional variation [49].

The principal limitations in the study are those inherent in spatial epidemiology. The finding of geographic hot spots of diffuse gastric cancers or $H$. pylori virulence factors may represent statistical artifacts albeit unlikely given the highly significant levels by two distinct methodologies. By necessity for the spatial analysis, cases outside of the western Honduras catchment areas and without aldea geocodes may have limited the sample size somewhat, and the ability to detect environmental associations. Exclusion of these populations would be unlikely to affect the principal spatial analysis in the defined catchment area.

\section{Conclusion}

Geospatial methods have identified a significant cluster of diffuse gastric adenocarcinoma patients, in a high incidence region of Central America. Investigation of potential germline mutations in this cluster of diffuse cancers is warranted. Further analyses to also study potential spatial patterns of bacterial and environmental risk factors may also be insightful.

\section{Abbreviations}

CA-4: Central America Four region: Honduras, Guatemala, Nicaragua, and El Salvador; EBV: Epstein-Barr virus; GIS: Geographic information systems;
HDGC: Hereditary diffuse gastric cancer; LMIC: Low/middle income country; TCGA: The Cancer Genome Atlas

\section{Acknowledgements}

We acknowledge the following individuals from the Hospital de Occidente, Honduras Ministry of Health, and Central American Medical Outreach for their contributions to this study: Eleazar E. Montalvan Sanchez MD, Aida A. Rodriguez Murillo MD, Daniela M. Montalvan Sanchez, MD, Lic. Lesby Castellanos, Samuel A. Urrutia-Argueta, MD

\section{Availability of data and material}

The datasets used and analyzed during the current study are available from the corresponding author on reasonable request. The datasets used to calculate crude incidence rates for GIS mapping are available from Instituto Nacional de Estadistica (INE) repository, https://www.ine.gob.hn/.

\section{Funding}

Funding for this study was obtained from Fogarty International Center and National Institute of Mental Health (R25 TW009337), National Cancer Institute (P01 CA028842, R01 CA190612, K07 CA125588, PAR-15-155, HHSN

261200800001E, P30 CA068485), and Vanderbilt Medical Scholars Program. The funders of the study had no role in study design, data collection, data analysis, data interpretation, or writing of the manuscript. DEO, CBC, RM, and DRM had full access to all the data in the study and take responsibility for the integrity of the data and the accuracy of the data analysis. DRM had final responsibility for the decision to submit for publication.

\section{Authors' contributions}

RLD, DRM and CBC designed and implemented the study. CBC, VE, ME designed and performed the geospatial analysis and interpretation. MPM and TW designed, performed and analyzed the H. pylori multiplex assay. PG, GT, and SMW interpreted and analyzed the pathology and genetic data. RM and DEO provided the overall statistical analysis and oversight. All authors with RP and KTW provided the critical review of the manuscript and approved the final manuscript. The study was presented in part as oral presentations the $\mathrm{NCl}$ Conference on Geospatial Approaches to Cancer Control and Population Sciences (September, 2016) and at Digestive Disease Week (May, 2017). All authors read and approved the final manuscript.

Ethics approval and consent to participate

Institutional review board approval for this study was obtained from committees at both the Hospital de Occidente de Honduras and Vanderbilt University Medical Center, and each subject provided written consent.

Consent for publication

Not applicable.

\section{Competing interests}

The authors declare that they have no competing interests.

\section{Publisher's Note}

Springer Nature remains neutral with regard to jurisdictional claims in published maps and institutional affiliations.

\section{Author details}

${ }^{1}$ Hospital de Occidente, Santa Rosa de Copan, Copan, Honduras. ${ }^{2}$ Office of Public Health Informatics \& Analytics, Tennessee Department of Public Health, Nashville, TN, USA. ${ }^{3}$ Vanderbilt Ingram Cancer Center (VICC), Vanderbilt University Medical Center, Nashville, USA. ${ }^{4}$ Division of Gastroenterology, Hepatology, and Nutrition, Vanderbilt University Medical Center, Nashville, USA. ${ }^{5}$ Carolina Population Center, University of North Carolina, Chapel Hill, USA. ${ }^{6}$ Division of Molecular Diagnostics of Oncogenic Infections, German Cancer Research Center (DKFZ), Heidelberg, Germany. ${ }^{7}$ Department of Population and Quantitative Health Sciences and Institute of Computational Biology, Case Western Reserve University, Cleveland, USA. ${ }^{8}$ Department of Pathology, University of North Carolina, Chapel Hill, USA. ${ }^{9}$ Department of Geography, University of North Carolina, Chapel Hill, USA ${ }^{10}$ Division of Gastroenterology and Hepatology, The University of Alabama at Birmingham (UAB), 1808 7th Avenue South, BDB 373, Birmingham, AL 35233, USA. 
Received: 4 May 2018 Accepted: 16 May 2019

Published online: 07 June 2019

\section{References}

1. Jemal A, Bray F, Center MM, Ferlay J, Ward E, Forman D. Global cancer statistics. CA Cancer J Clin. 2011;61(2):69-90.

2. Ferlay J, Soerjomataram I, Dikshit R, et al. Cancer incidence and mortality worldwide: sources, methods and major patterns in GLOBOCAN 2012. Int $J$ Cancer. 2015;136(5):E359-86.

3. Plummer M, de Martel C, Vignat J, Ferlay J, Bray F, Franceschi S. Global burden of cancers attributable to infections in 2012: a synthetic analysis. Lancet Glob Health. 2016;4(9):e609-16.

4. Bray F, Jemal A, Grey N, Ferlay J, Forman D. Global cancer transitions according to the human development index (2008-2030): a populationbased study. Lancet Oncol. 2012;13(8):790-801.

5. Parkin DM, Bray F, Ferlay J, Pisani P. Global cancer statistics, 2002. CA Cancer J Clin. 2005:55(2):74-108.

6. Torres J, Correa P, Ferreccio C, et al. Gastric cancer incidence and mortality is associated with altitude in the mountainous regions of pacific Latin America. Cancer Causes Control. 2013;24(2):249-56.

7. Montero-Oleas N, Núñez-González S, Simancas-Racines D. The remarkable geographical pattern of gastric cancer mortality in Ecuador. Cancer Epidemiol. 2017;51:92-7.

8. Kodaman N, Pazos A, Schneider BG, Piazuelo MB, Mera R, Sobota RS, Sicinschi LA, Shaffer CL, Romero-Gallo J, de Sablet T, Harder RH. Human and helicobacter pylori coevolution shapes the risk of gastric disease. Proc Natl Acad Sci. 2014;111(4):1455-60.

9. Falush D, Kraft C, Taylor NS, Correa P, Fox JG, Achtman M, Suerbaum S. Recombination and mutation during long-term gastric colonization by helicobacter pylori: estimates of clock rates, recombination size, and minimal age. Proc Natl Acad Sci. 2001;98(26):15056-61.

10. Tavera G, Morgan DR, Williams SM. Tipping the scale towards gastric disease: a host-pathogen genomic mismatch? Curr Genet Med Rep. 2018; 6(4):199-207.

11. Feldman RA, Eccersley AJP, Hardie JM. Epidemiology of Helicobacter pylori-acquisition, transmission, population prevalence and disease-toinfection rate. Br Med Bull. 1998:54:39-53.

12. Plummer M, Franceschi S, Vignat J, Forman D, de Martel C. Global burden of gastric cancer attributable to helicobacter pylori. Int J Cancer. 2015;136(2):487-90.

13. Polk DB, Peek RM Jr. Helicobacter pylori: gastric cancer and beyond. Nat Rev Cancer. 2010;10(6):403

14. Yong X, Tang B, Li BS, Xie R, Hu CJ, Luo G, Qin Y, Dong H, Yang SM. Helicobacter pylori virulence factor CagA promotes tumorigenesis of gastric cancer via multiple signaling pathways. Cell Commun Signal. 2015;13(1):30.

15. Backert S, Blaser MJ. The role of CagA in the gastric biology of helicobacter pylori. Cancer Res. 2016;76(14):4028-31.

16. Correa P, Houghton J. Carcinogenesis of helicobacter pylori. Gastroenterology. 2007;133(2):659-72.

17. Lauren P. The two histological main types of gastric carcinoma: diffuse and socalled intestinal-type carcinoma. Acta Pathol Microbiol Scand. 1965;64:31-49.

18. Rugge M, Fassan M, Graham DY. Epidemiology of gastric cancer. In: Gastric Cancer. Cham: Springer; 2015. p. 23-34.

19. Cancer Genome Atlas Research Network. Comprehensive molecular characterization of gastric adenocarcinoma. Nature. 2014;513(7517):202.

20. Kupfer SS. Gaining ground in the genetics of gastric Cancer. Gastroenterology. 2017;152(5):926-8.

21. Sahasrabudhe R, Lott P, Bohorquez M, Toal T, Estrada AP, Suarez JJ, BreaFernández A, Cameselle-Teijeiro J, Pinto C, Ramos I, Mantilla A. Germline mutations in PALB2, BRCA1, and RAD51C, which regulate DNA recombination repair, in patients with gastric cancer. Gastroenterology. 2017;152(5):983-6.

22. Corral JE, Hurtado JJ, Domínguez RL, de Cuéllar MV, Cruz CB, Morgan DR. The descriptive epidemiology of gastric cancer in Central America and comparison with United States Hispanic populations. J Gastrointest Cancer 2015:46(1):21-8.

23. Pickle LW, Szczur M, Lewis DR, Stinchcomb DG. The crossroads of GIS and health information: a workshop on developing a research agenda to improve cancer control. Int J Health Geogr. 2006;5(1):51.

24. Aneja S, Gross CP, Soulos PR, Yu JB. Geographical information systems: applications and limitations in oncology research. Oncology. 2011;25(12):1221-5.
25. Amani F, Ahari SS, Barzegari S, Hassanlouei B, Sadrkabir M, Farzaneh E. Analysis of relationships between altitude and distance from volcano with stomach Cancer incidence using a geographic information system. Asian Pac J Cancer Prev. 2015;16(16):6889-94.

26. Ruiz EF, Torres-Roman JS, Servan SA, et al. Trends and geographic pattern of stomach cancer mortality in Peru. Cancer Epidemiol. 2019;58:193-8.

27. Piñeros $M$, Frech $S$, Frazier $L$, al e. Advancing reliable data for Cancer control in the Central America four region. J Glob Oncol. 2018;(4):1-11.

28. Dominguez RL, Crockett SD, Lund $J$, et al. Gastric cancer incidence estimation in a resource-limited nation: use of endoscopy registry methodology. Cancer Causes Control. 2013;24(2):233-9.

29. Greenberg ER, Anderson GL, Morgan DR, et al. 14-day triple, 5-day concomitant, and 10-day sequential therapies for helicobacter pylori infection in seven Latin American sites: a randomised trial. Lancet. 2011; 378(9790):507-14.

30. Honduras National Census Data. National Statistics Institute (INE), Honduras 2013. https://www.ine.gob.hn/. Accessed 1 Feb 2018.

31. Michel A, Waterboer T, Kist M, Pawlita M. Helicobacter pylori multiplex serology. Helicobacter. 2009;14(6):525-35.

32. Waterboer T, Sehr $\mathrm{P}$, Michael KM, et al. Multiplex human papillomavirus serology based on in situ-purified glutathione s-transferase fusion proteins. Clin Chem. 2005:51(10):1845-53.

33. Waterboer T, Sehr P, Pawlita M. Suppression of non-specific binding in serological luminex assays. J Immunol Methods. 2006:309(1-2):200-4.

34. Gao L, Weck MN, Michel A, Pawlita M, Brenner H. Association between chronic atrophic gastritis and serum antibodies to 15 helicobacter pylori proteins measured by multiplex serology. Cancer Res. 2009;69(7):2973-80.

35. Epplein $M$, Zheng $W$, Xiang $Y B$, et al. Prospective study of helicobacter pylori biomarkers for gastric cancer risk among chinese men. Cancer Epidemiol Biomark Prev. 2012;21(12):2185-92.

36. Song H, Michel A, Nyren O, Ekstrom AM, Pawlita M, Ye W. A CagA-independent cluster of antigens related to the risk of noncardia gastric cancer: associations between helicobacter pylori antibodies and gastric adenocarcinoma explored by multiplex serology. Int J Cancer. 2014;134(12):2942-50.

37. Ord JK, Getis A. Local spatial autocorrelation statistics: distributional issues and an application. Geogr Anal. 1995;27(4):286-306.

38. Kulldorff M. A spatial scan statistic. Communications in Statistics-Theory and Methods. 1997;26(6):1481-96.

39. van der Post RS, Vogelaar IP, Carneiro F, et al. Hereditary diffuse gastric cancer: updated clinical guidelines with an emphasis on germline $\mathrm{CDH} 1$ mutation carriers. J Med Genet. 2015;52(6):361-74.

40. Hansford S, Kaaurah P, Li-Chang H, et al. Hereditary diffuse gastric Cancer syndrome: CDH1 mutations and beyond. JAMA Oncol. 2015;1(1):23-32.

41. Ford JM. Hereditary gastric Cancer: an update at 15 years. JAMA Oncol. 2015;1(1):16-8.

42. Slavin T, Neuhausen SL, Rybak C, et al. Genetic gastric Cancer susceptibility in the international clinical Cancer genomics community research Network. Cancer Genet. 2017:216-217:111-9.

43. Kaurah P, MacMillan A, Boyd N, Senz J, De Luca A, Chun N, Suriano G, Zaor S, Van Manen L, Gilpin C, Nikkel S. Founder and recurrent CDH1 mutations in families with hereditary diffuse gastric cancer. JAMA. 2007;297(21):2360-72.

44. Guilford P, Hopkins J, Harraway J, McLeod M. E-cadherin germline mutations in familial gastric cancer. Nature. 1998:392(6674):402.

45. Gigek CO, Calcagno DQ, Rasmussen LT, Santos LC, Leal MF, Wisnieski F, Burbano RR, Lourenco LG, Lopes-Filho GJ, Smith MA. Genetic variants in gastric cancer: risks and clinical implications. Exp Mol Pathol. 2017;103(1):101-11.

46. Tang W, Morgan DR, Meyers DO, et al. Epstein-barr virus infected gastric adenocarcinoma expresses latent and lytic viral transcripts and has a distinct human gene expression profile. Infect Agent Cancer. 2012;7:21.

47. Borozan I, Zapatka M, Frappier L, Ferretti V. Analysis of Epstein-Barr virus genomes and expression profiles in gastric adenocarcinoma. J Virol. 2018; 92(2):e01239-17.

48. Dheekollu J, Malecka K, Wiedmer A, Delecluse HJ, Chiang AK, Altieri DC, Messick TE, Lieberman PM. Carcinoma-risk variant of EBNA1 deregulates Epstein-Barr virus episomal latency. Oncotarget. 2017:8(5):7248.

49. Singh S, Jha HC. Status of Epstein-Barr virus coinfection with Helicobacter pylori in gastric cancer. J Oncol. 2017;2017:3456264. 